\title{
A moving direction prediction-assisted handover scheme in LTE networks
}

\author{
Hsiu-Lang Wang ${ }^{1}$, Shang-Juh Kao ${ }^{1}$, Chung-Yi Hsiao ${ }^{1}$ and Fu-Min Chang ${ }^{2 *}$
}

\begin{abstract}
The 3rd Generation Partnership Project (3GPP) Long Term Evolution (LTE) defines a wireless network standard for high packet transmission rate and low packet latency provisions. Handover is one of the important features for helping user equipments (UEs) to roam between LTE networks. However, LTE networks adapt a make-before-break handover procedure, which may cause a brief disconnection, therefore results in the packet transmission delay and packet loss problems. In this paper, we propose a moving direction prediction-assisted handover scheme for LTE networks to lower the number of handovers. We first track the location of user equipments (UEs) to predict their moving direction. By referencing previous locations, the next moving direction of UEs is estimated with the cosine function in order to determine the candidate E-UTRAN NodeBs (eNBs) for handover. Then, a target eNB is selected from the candidate eNBs through an angle-based dynamic weight adjustment scheme. By selecting a proper target eNB for handover, thus the quality of network transmission can be enhanced. Simulation results demonstrate the ability of the proposed scheme in reducing $17 \%$ average handover times, compared with the standard handover procedure, thereby reducing $12 \%$ average number of packet loss and 5\% average packet delay time.
\end{abstract}

Keywords: Handover; Moving direction prediction; LTE networks

\section{Introduction}

To meet the increasing demand of wireless data services, the 3rd Generation Partnership Project (3GPP) has proposed UMTS Terrestrial Radio Access Network (UTRAN) Long Term Evolution (LTE) [1]. LTE networks provide high-speed communication within uplink and downlink, as well as aiming to provide more capacity along with less network complexity and low installation and maintenance cost [2]. Two different radio access mechanisms are used in LTE networks, orthogonal frequency-division multiple access (OFDMA) is used for downlink and singlecarrier frequency-division multiple access (SC-FDMA) is used for uplink [3,4]. OFDMA provides high spectral efficiency which is very immune to interference and reduces computation complexity in the terminal within larger bandwidths [5]. SC-FDMA has lower peak to average power ratio (PAPR) provisions, which leads toward longer battery use as well as larger radio coverage [6]. Another advantage of using LTE networks is its

\footnotetext{
*Correspondence: fmchang@cyut.edu.tw

2 Department of Finance, Chaoyang University of Technology, 168, Jifeng E.

Rd., Wufeng District, 41349 Taichung, Taiwan
}

Full list of author information is available at the end of the article capability to switch back to older legacy systems such as Wideband Code Division Multiple Access (WCDMA), when user equipment (UE) gets out of coverage from an LTE network [7].

As shown in Figure 1, the LTE network architecture consists of evolved NodeBs (eNBs), mobility management entity (MME), and system architecture evolution gateways (S-GW) [5]. The eNBs are connected to the MME/SGW by the S1 interface, and they are interconnected by the X2 interface. Handover is one of the important features within LTE networks, which assists UEs to roam within the signal coverage of eNBs. The necessary handover information is exchanged between eNodeBs via the $\mathrm{X} 2$ interface. In LTE networks, hard handover has been considered to minimize the radio resource requirement [8]. Because hard handover is more sensitive to radio link failure than soft handover, it is required to minimize the number of handovers for maintaining the given quality of service $(\mathrm{Q} o \mathrm{~S})$ within a communication link. Hence, an efficient handover mechanism with lower number of handovers is necessary for LTE networks [9].

In LTE networks, the UE performs several downlink radio channel measurements on both serving cell and

\section{Springer}

(c) 2014 Wang et al: licensee Springer. This is an Open Access article distributed under the terms of the Creative Commons Attribution License (http://creativecommons.org/licenses/by/4.0), which permits unrestricted use, distribution, and reproduction in any medium, provided the original work is properly credited. 


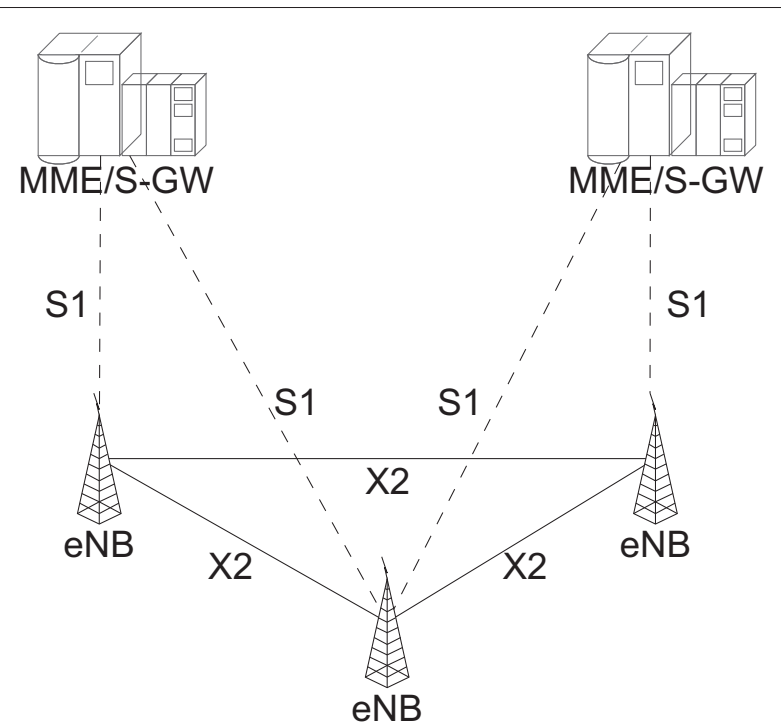

Figure 1 E-UTRAN architecture. The LTE network architecture consists of evolved NodeBs (eNBs), mobility management entity (MME), and system architecture evolution gateways (S-GW).

neighboring cells by using reference symbols (RS) [10]. Those measurements are used to quantify network performance. Handover execution may occur mainly due to poor cell coverage or poor QoS level. The cell coverage is monitored using the reference signal received power (RSRP) from serving cell and neighboring cells. On the other hand, QoS level is measured using the reference symbol received quality (RSRQ) and other parameters $[7,11]$. The RSRP and RSRQ are measured by UEs during a designated measurement interval. In case of coveragebased handover, if the RSRP of one of the UE neighboring cells is greater than the RSRP of the serving cell plus a designated hysteresis value for at least a time-to-trigger period (TTT), the handover procedure triggering occurs [5]. Once triggering conditions are met, the UE sends the measurement report back to its serving eNB, indicating the triggering event and the target cell with the highest RSRP level when compared with the current serving cell. Based on the received measurement report, the serving eNB starts the handover preparation phase. In contrary, if RSRP in the serving cell becomes higher again than that in the target cell, during TTT period, the handover procedure would not be executed. LTE femtocell architecture has been proposed to gain a better indoor radio signal quality with low-cost deployment. It offers plug and play for configuration, low deployment cost, and traffic offload from the macrocell $[12,13]$. However, the distributed deployment scheme results in signal interference $[14,15]$. This can significantly impact on the transmission power measurement of UEs, which consequently impact the handover decision.
The handover procedure takes extra time and signaling overhead to synchronize and communicate between UE and eNB [16], in order to maintain the data connection. Due to the unavoidable handover delay [17], uninterrupted communication can be hardly accomplished, which may seriously affect the performance of a real-time application. Moreover, there is a risk of the handover fail which may result in a radio link failure [18]. The radio link failure rate can be increased because of delayed handover execution during TTT period. This phenomenon has been aggravated by unnecessary handovers, which has adverse effect on the system performance [19]. Too many unnecessary handovers may also result in signaling overhead $[8,20]$. Therefore, to lower the number of handover, we propose an improved handover mechanism by predicting the moving direction of UEs in this work. By referencing the previous locations of UEs, the next moving direction for each UE is estimated with the cosine function in order to determine the candidate eNBs for handover. An accurately target cell for handover is selected from the numbers of filtered candidate eNBs through an anglebased dynamic weight adjustment scheme. The number of handover is expected to be reduced through an accurately target cell selection, thereby also reducing packet loss and packet delay time.

This paper proceeds as follows: the 'Related work' section describes the researches on prediction-based handover algorithm and TTT. The proposed mechanism is described in 'A moving direction prediction-assisted handover mechanism' section. The simulation results are shown in the 'Simulation and results' section. Finally, the 'Conclusions' section concludes the paper and summarized the future work.

\section{Related work}

The handover procedure is required when UE moves between eNBs, which leads to extra handover cost to ensure the network connection quality. In [21], wireless ad hoc networks have been introduced to assist the handover procedure of the UE. Before UE handovers to a target eNB, a relay node is selected from wireless ad hoc networks. All handover-related information of the UE has been transferred to the target eNB via the selected relay node in advance. Fast handover can be achieved with the cost of increasing the loading of the relay node and may have security issues.

For the researches on TTT, in [22], the authors examined the handover performance by using various TTT values in LTE networks, depending on UE speeds and cell configurations within an allowable radio link failure rate. In the first step, the adaptive TTT values for each UE speeds were selected in macro-macro handover and macro-pico handover scenarios. And then, the authors fitted the curve by using the result of selected 
TTT values for both neighboring cell configurations. From the TTT curve, the authors suggested the criteria for grouping UE speeds and the proper TTT values in accordance with the cell configurations. The simulation results showed that the performance was comparable with that of the case when applying the adaptive TTT values for an arbitrary UE speed, and the performance is significantly improved as compared to that of the cases when a fixed TTT value was applied in both cell type configurations.

Different research approaches try to use movement predictions as an addition to classical handover preparation and triggers. In [23], the authors proposed a mobility management technique which uses simple handover prediction based on cross-layer architecture. Their prediction technique uses both simple moving average for inertial movements and simple mobility pattern matching for non-inertial movements. They also introduced a new reporting event for UE's measurement reports and designed a handover prediction algorithm for eNodeB's handover preparation. Although they have outperformed in terms of number of handovers and rate of pingpong handover, a simple knowledge database is required to overcome the unnecessary handover issues, thereby increases the complex of operations and extra cost.

In [24], the authors proposed a handover approach with a simple handover prediction based on acts of users of mobile history. They used the user mobility database and simple mobility pattern matching as well. A valid update database approach is proposed to ensure the database time in tracking the user mobility actions. However, both approaches reduce the evaluating cost of more candidate target eNBs, while increase the cost of mobility database. When the current move pattern of the user is regular, the candidate base station is filtered out in advance for a handover preparation, which can reduce number of handovers. However, in order to reduce the evaluating cost for eNBs, the cost of mobility database is increased.

In [25], a method for minimizing unnecessary handovers in heterogeneous wireless networks has been proposed. When a macro user moves into a micro cell, the traveling distance is estimated based on the signal measurements and the velocity of the user. The traveling distance is compared with a predefined threshold, in order to determine the handover decision. The proposed scheme has outperformed in minimizing the probability of handover failures and unnecessary handovers when compared with conventional methods. However, the handover in homogeneous networks is out of their scope.

\section{A moving direction prediction-assisted handover mechanism}

As UEs move around the signal coverage of LTE networks, the terrain may influence the received signal power, especially when UEs locate far away its serving eNB. To maintain a certain communication quality, eNBs will inform UEs, which have the weaker feedback radio signal strength, to trigger handover procedures. The handover procedures in LTE networks can be divided into three distinct phases: the preparation, execution, and completion phases. Before UEs enter in handover preparation phase, UEs have feedback measurement reports to its serving eNB periodically. The measurement reports include the signal strength of its serving and neighbor eNBs. The neighbor eNBs form as candidate eNBs for handover, and it takes time to select a target eNB for handover. The make-before-break handover strategy may result in disconnection and data loss, where a real-time service impacts. Therefore, a direction prediction scheme and a dynamic weight adjustment scheme are proposed to reduce the number of handovers.

\section{Direction prediction scheme}

In the proposed moving direction prediction scheme, we adopt the Global Positioning System (GPS) to identify the position of each eNBS and UE [26]. The GPS is a satellite navigation system and provides accurate positioning, velocity, and precision standard time for most of the Earth's surface area. Since most of the smartphones have been already embedded with the GPS service, it is convenient for devices to acquire its position. The position of each eNB also can be measured by GPS, because the deployment of each eNB is planned and designed by network operators. Therefore, with the help of GPS, the position of each eNB and UE can be identified separately for tracing.

Since the moving direction of a UE may be toward a specific group of neighbor eNBs, the moving path of the UE has to be recorded at first for its direction prediction. The position of the UE can be retrieved from the GPS and represented by $P_{1}, P_{2}, P_{3}$, where $P_{3}$ is the position at the current time stamp, $P_{2}$ is at the previous time stamp, and $P_{1}$ is the position before the previous time stamp as shown in Figure 2. Recalling the trigonometric functions and inner product formula, giving three points, a $\theta$ angle is obtained by a function of angle calculator as shown in Algorithm 1.

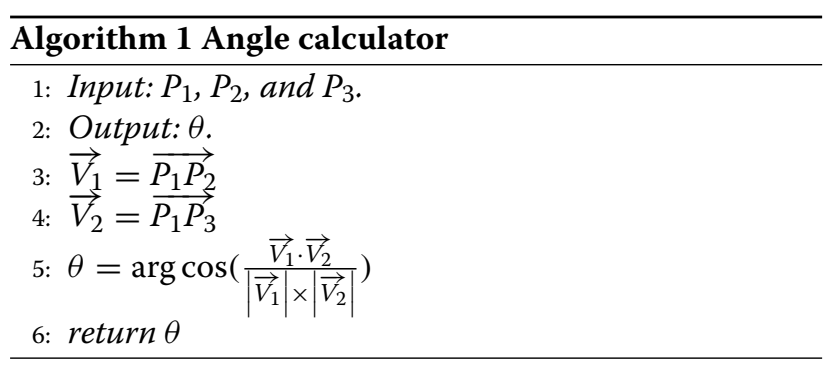




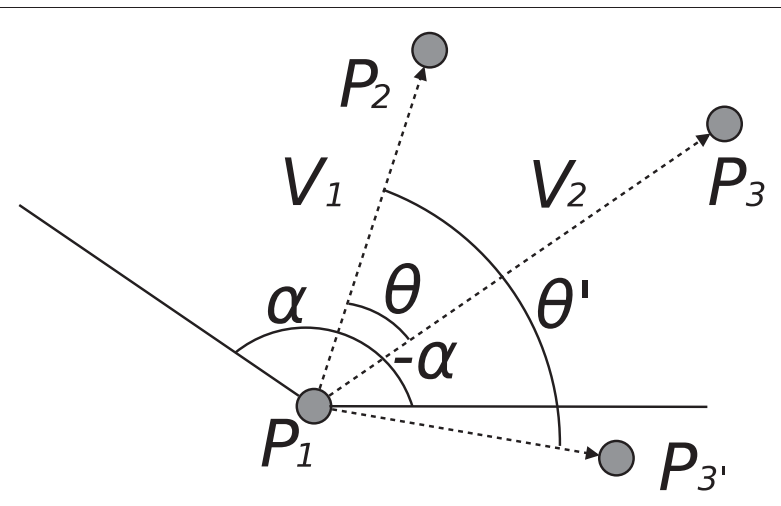

Figure 2 Example of a moving direction prediction. The position of the UE can be retrieved from the GPS and represented by $P_{1}, P_{2}, P_{3}$.

We illustrate the moving direction prediction scheme with Figure 2 as follows. $V_{1}$ is the vector of $P_{1} P_{2}, V_{2}$ is the vector of $P_{1} P_{3}$, and $\theta$ is the angle of $\angle P_{2} P_{1} P_{3}$. The $\theta$ is calculated along with the moving path of a UE. It is used to determine if the UE moves in a specific direction.

Initially, positions $P_{1}$ and $P_{2}$ of a UE are obtained from GPS. Each time the new position $P_{3}$ of the UE is updated to its associated eNB, the angle calculator is called with $P_{1}, P_{2}, P_{3}$ parameters to find out a $\theta$ value and return. The $\alpha$ is an angle offset value, which is used to determine if a new location, $P_{3}$, located within another predefined angle offset range, $\pm \alpha . \theta$ is compared with $\alpha$ value to check if the moving directions of $P_{1} P_{3}$ and $P_{1} P_{2}$ are the same. If it is, then the eNBs, which are located with the range of $\alpha$, that will be considered as candidate eNBs. Since each eNB is assumed to be equipped with three antennas to provide its signal coverage. Each antenna can provide signal coverage with the range of $120^{\circ}$ angle and form as a cellular network. Therefore, we defined $\alpha$ has $\pm 60^{\circ}$ angle offset for a main direction boundary of a UE in this study; the moving path within the range of $120^{\circ}$ angle ( $\pm \alpha$ degree based on $P_{1} P_{2}$ ) totally is determined as the same direction of $P_{1} P_{2}$. In short, the moving path $P_{1} P_{3}$ has the same direction with $P_{1} P_{2}$ in the case of the angle $\angle P_{3} P_{1} P_{2}$ less than $|\alpha|$ as shown in Figure 2 .

In the case of $P_{1} P_{3}^{\prime}$ which has a different moving direction with $P_{1} P_{2}$, where $\theta^{\prime}$ is not less than $|\alpha|$, the main moving direction of the UE is re-defined. The dynamic weights of candidate eNBs, as described in the next subsection, are reset to zero. Then, $P_{2}$ is transferred to $P_{1}$, and $P_{3}^{\prime}$ is transferred to $P_{2}$. As a result, the new direction of $P_{1} P_{2}$ becomes a new main moving direction of the UE.

\section{Dynamic weight adjustment scheme}

In the case of $P_{1} P_{3}$ which has the same moving direction with $P_{1} P_{2}$, the candidate eNBs are required to be filtered from all neighbor eNBs. In order to filter the candidate
eNBs, the $\pm \alpha$ degree is defined based on $P_{2} P_{3}$. For each neighbor eNB of the UE, the angle offset value, $\theta_{\mathrm{eNB}}$, is calculated. The $\theta_{\mathrm{eNB}}$ is compared with $|\alpha|$; if its value is less than $|\alpha|$, the eNB is designated as a candidate eNB. As shown in Figure 3, $\mathrm{eNB}_{0}$ is a serving eNB of the UE. After UE moves to $P_{3}$ from $P_{1}$ via $P_{2}, P_{3}$ is determined within the main moving direction of $P_{1} P_{2}$. The $\theta_{\mathrm{eNBs}}$ for $\mathrm{eNB}_{1}$, $\mathrm{eNB}_{2}$, and $\mathrm{eNB}_{3}$ are calculated. Only $\mathrm{eNB}_{2}$ and $\mathrm{eNB}_{3}$ are filtered and designated as candidate eNBs for the UE.

Since too many candidate eNBs will increase the probability of unnecessary handover, UEs also waste power to do unnecessary measurement reports from these filtered candidate eNBs. Therefore, once the main moving direction of each UE is predicted and the candidate eNBs is filtered, the target eNB is selected with a dynamic weight adjustment scheme as shown in Algorithm 2.

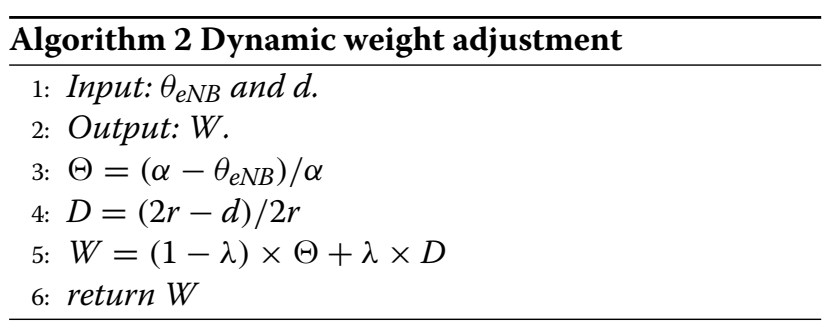

Both $\theta_{\mathrm{eNB}}$ and distance $(d)$ between UE and candidate eNBs are taken into consideration for a weight $(W)$ calculation. The smaller $\theta_{\mathrm{eNB}}$ and distance indicates that the $\mathrm{UE}$ is approaching to that candidate eNB. Since the eNB with $\theta_{\mathrm{eNB}}$ less than $\pm \alpha$ is filtered as a candidate eNB, the $\theta_{\mathrm{eNB}}$ is normalized with $\alpha$ and stored in $\Theta$. The distance is normalized with $r$, where $r$ is a transmission range of the $\mathrm{eNB}$, and stored in $D$. A variable $\lambda$, where $0 \leq \lambda \leq 1$, is adopted to control the angle offset and distance value. $W$ is then returned for a target $\mathrm{eNB}$ selection.

\section{Moving direction prediction-assisted handover procedure}

Together with the previous two subsections, a direction prediction-assisted handover procedure is presented in Algorithm 3. The algorithm executes on each eNB. Initially, $P$ and $Q$ are two set of eNBs, where $P$ contains all eNBs but $Q$ is empty. For a UE-associated eNB, it is assumed that $P_{1}$ and $P_{2}$ of the UE are already reported to eNB. When serving eNB receives $P_{3}$ information in line 3 which is reported by the UE, it calls the function of angle calculator in line 4 with parameters $P_{1}, P_{2}$, and $P_{3}$ and gets the returned $\theta$ value. If $\theta$ is less or equal $|\alpha|$ in line 5 , it indicates that $P_{3}$ locates within the main moving direction of $P_{1} P_{2}$. The serving eNB then retrieves all its neighbor eNBs from $P$ sequentially in line 6 and calls the function of angle calculator again with parameters $P_{2}, P_{3}$, and $P_{\mathrm{eNB}}$ in line 7, in order to get the $\theta_{\mathrm{eNB}}$. If $\theta_{\mathrm{eNB}}$ is less or equal $|\alpha|$ 


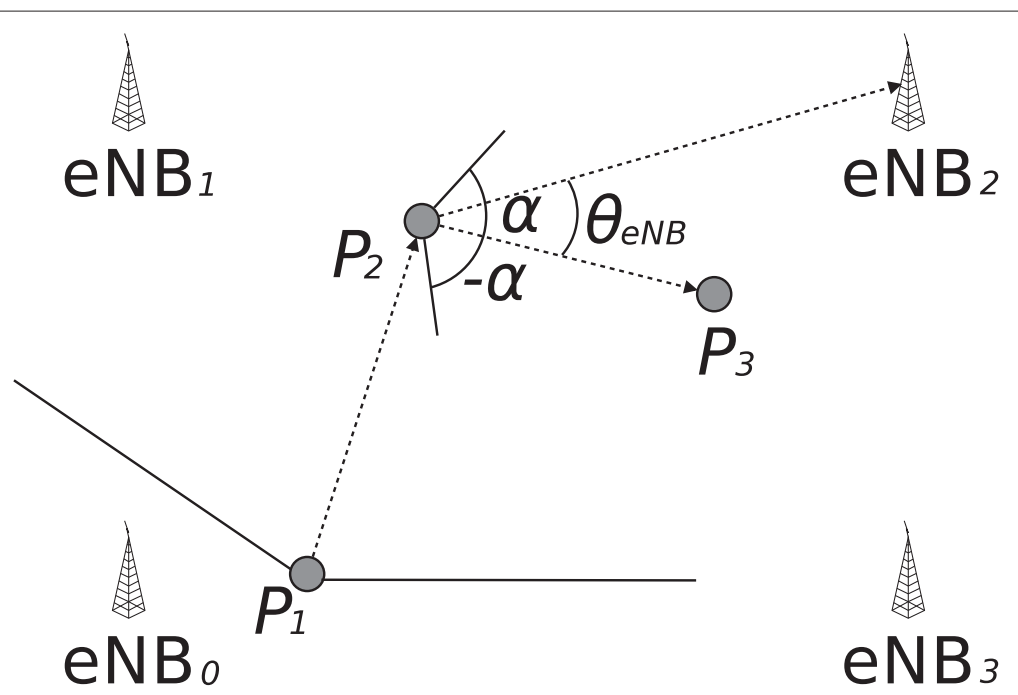

Figure 3 Example of a candidate eNB selection. The $\theta_{\mathrm{eNB}}$ is compared with $|\alpha|$; if its value is less than $|\alpha|$, the eNB is designated as a candidate eNB.

in line 8, it indicates that the eNB is a candidate and put it into $Q$ set in line 9 .

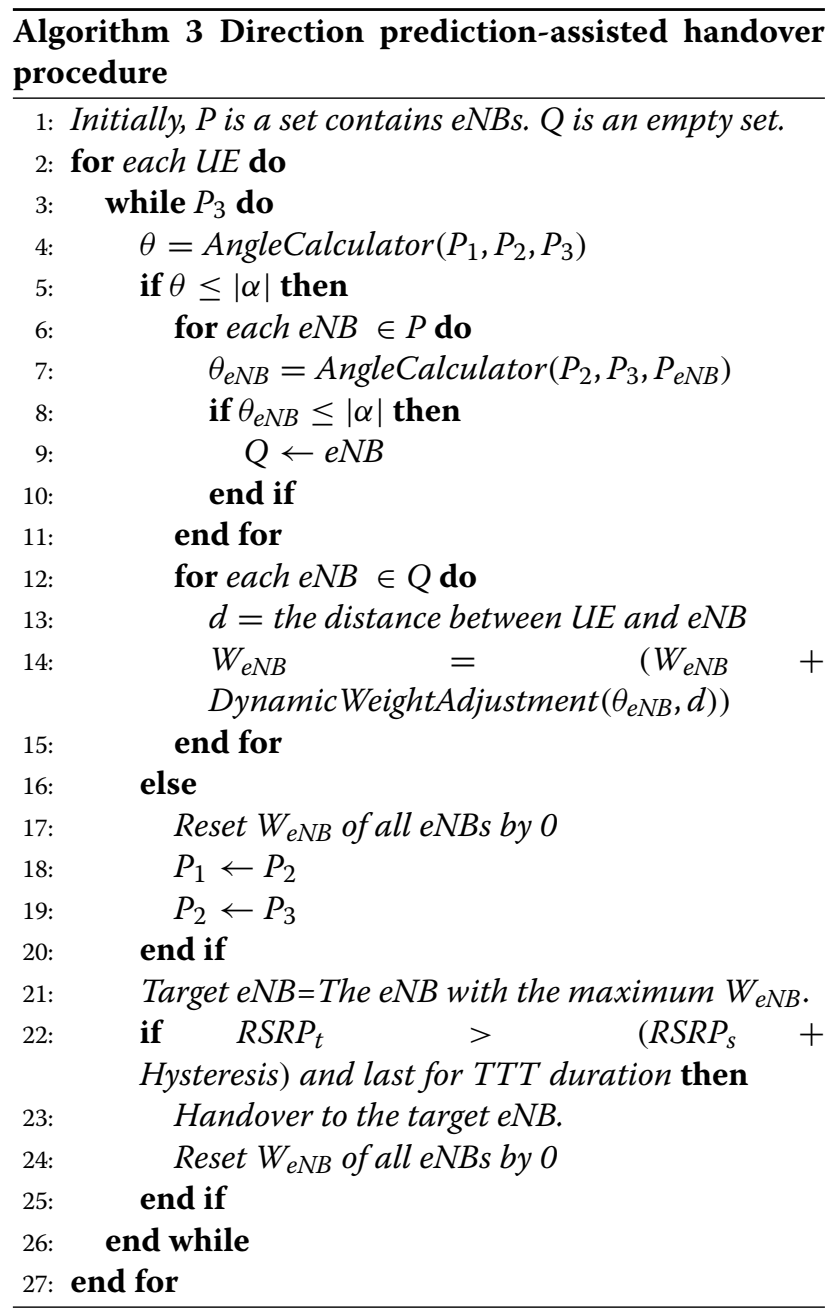

After the for loop in line 11, $Q$ contains all candidate eNBs. The candidate eNBs then are retrieved from $Q$ sequentially in line 12 , and the distance between UE and each candidate eNB is calculated and stored in a $d$ variable in line 13. The function of dynamic weight adjustment with parameters $\theta_{\mathrm{eNB}}$ and $d$ is called in line 14. It returns $W$ value which is summarized with its previous $W_{\mathrm{eNB}}$ value. $W_{\mathrm{eNB}}$ variable stores the weight for each candidate eNB.

If the condition of line 5 is not true, it indicates that the UE moves out of the main direction of $P_{1} P_{2}$. Then, $W_{\mathrm{eNB}}$ resets to zero for each candidate eNB in line 17. The location of $P_{2}$ and $P_{3}$ then are transferred to $P_{1}$ and $P_{2}$, respectively, in lines 18 to 19 . The obtained $P_{1} P_{2}$ will become a new moving direction.

The target eNB is selected from the candidate eNBs which has the maximum $W_{\mathrm{eNB}}$ in line 21 . The UE continually measures and reports the signal strength from its serving eNB and target eNB. If the radio signal strength of the target eNB is larger than the serving eNB plus a predefined hysteresis value, and last for TTT duration in line 22, the handover procedure is activated in line 23. All $W_{\mathrm{eNB}}$ are set to zero since the UE is expected to be handover to a target eNB in line 24 .

\section{Simulation and results}

To verify the proposed mechanism, a LTE-sim downlink system level simulation (SL simulator) [27] was adopted to evaluate the performance. The average number of measurement report, average number of handover, packet loss rate, and average transmission delay of handovers are evaluated with various velocities of UEs. We first introduce the simulation environment and related parameters, and then the simulation results are presented. 


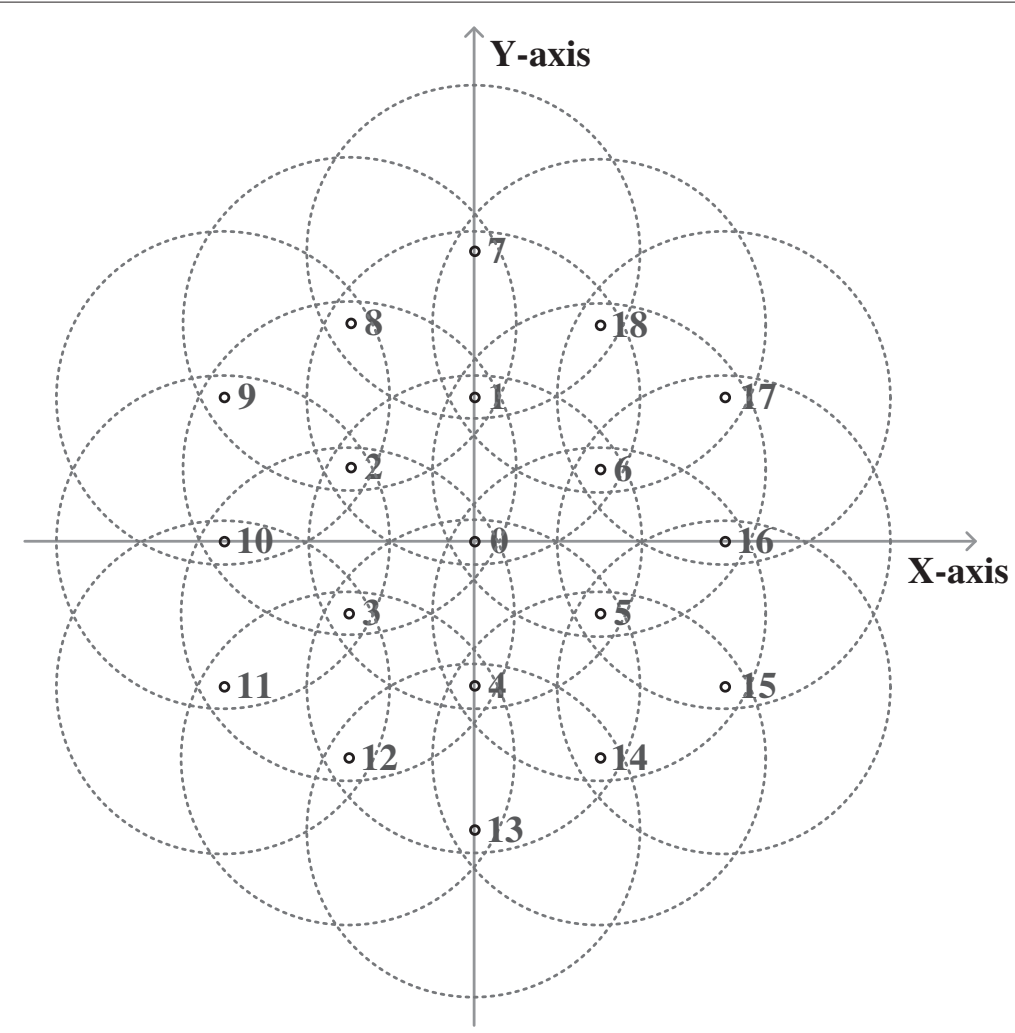

Figure 4 Network topology of simulation. The network topology of simulation consists of 19 eNBs indexed from 0 to 18.

\section{Simulation environment and parameters}

As shown in Figure 4, the network topology of simulation consists of 19 eNBs indexed from 0 to 18 , where each eNB has three sectors and each sector has one UE associated. There are 57 UEs totally deployed. Some main simulation parameters are listed in Table 1, which are referenced from [28]. UEs move randomly with predefined speed in $30 \mathrm{~s}$ of simulation time. The velocities of UEs are configured into $3,30,120$, and $150 \mathrm{~km} / \mathrm{h}$.

When UE enters into LTE networks, it would measure the signal strength of serving eNB and neighbor eNBs. The measurement report will feed back to its serving eNB to select a target eNB for handover. Too many frequently signal measurements would waste the power of the UE. When UE roams within LTE networks, hard handover is required to keep data connection, which may result in packet loss and transmission delay. We compare the performance metrics of the average number of measurement report, average number of handover, packet loss rate, and average transmission delay for standard handover scheme. The performance metrics are listed in the following equation:

$$
\text { Average number of measurement reports }=\frac{M}{N \times T} .
$$

Average number of handovers $=\frac{H}{N \times T}$.

Number of packet loss $=\frac{P_{s}-P_{r}}{T}$.

Average transmission delay $=\frac{\sum_{i=1}^{P_{r}} T_{i}^{r}-T_{i}^{s}}{P_{r}}$.

\section{Table 1 Main simulation parameters}

\begin{tabular}{ll}
\hline Parameters & Value \\
\hline Frequency & $2 \mathrm{GHz}$ \\
Bandwidth & $5 \mathrm{MHz}$ \\
Thermal noise density & $-174 \mathrm{dBm} / \mathrm{Hz}$ \\
Receiver noise value & $9 \mathrm{~dB}$ \\
eNB's transmission power & $46 \mathrm{dBm}$ \\
Resource blocks per eNB & 25 \\
Bandwidth per eNB & $180 \mathrm{kHz}$ \\
TTl (subframe duration) & $1 \mathrm{~ms}$ \\
Numbers of UE & 57 \\
Cell layout & $19 \mathrm{sites}, 3$ sector per site \\
Simulation time & $20 \mathrm{~s}$ \\
UE speed & $3,30,120$, and $150 \mathrm{~km} / \mathrm{h}$ \\
Mobility model & Random waypoint \\
Scheduling strategy & Proportional fair \\
\hline
\end{tabular}




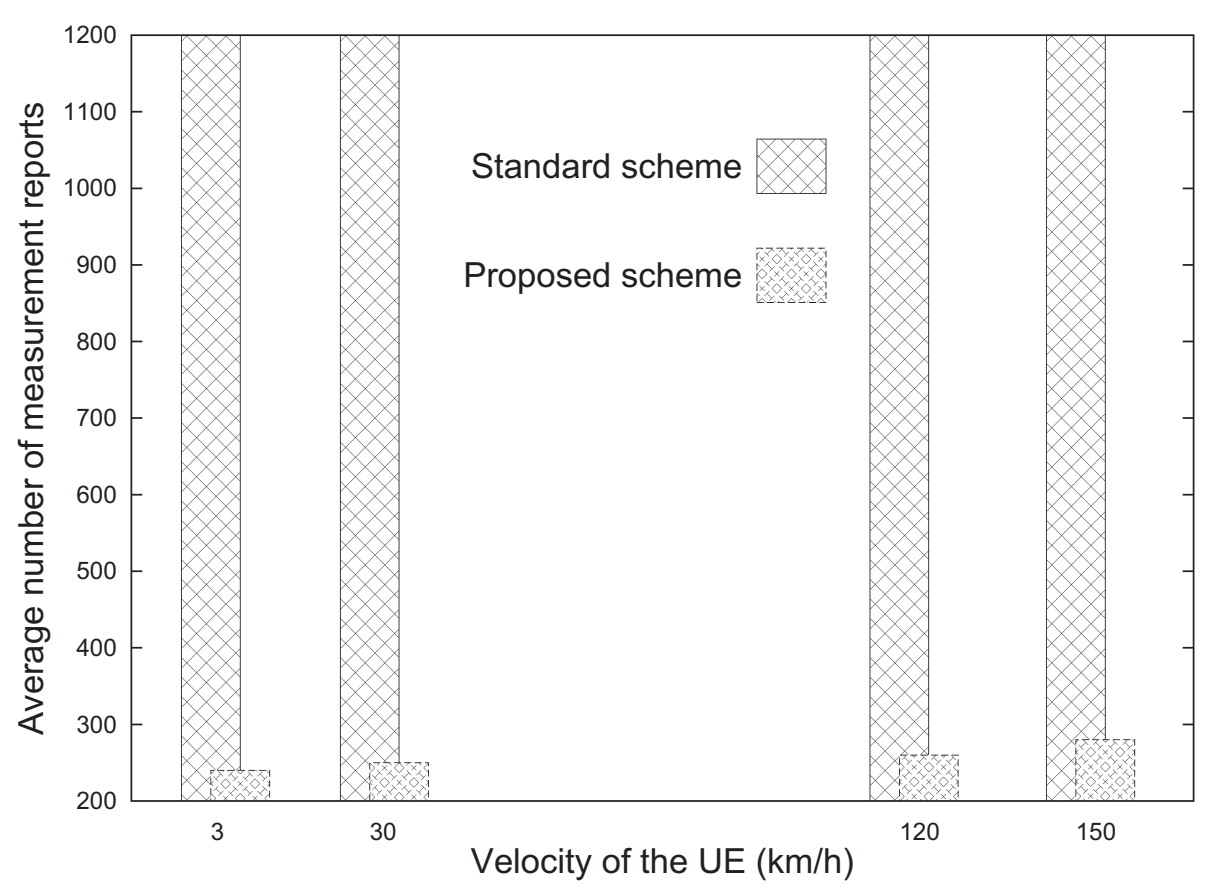

Figure 5 The average number of measurement reports for different handover schemes. UE periodically measures the single strength of serving and target eNBs with our proposed scheme, instead of measuring all its neighbor eNBs with the standard scheme.

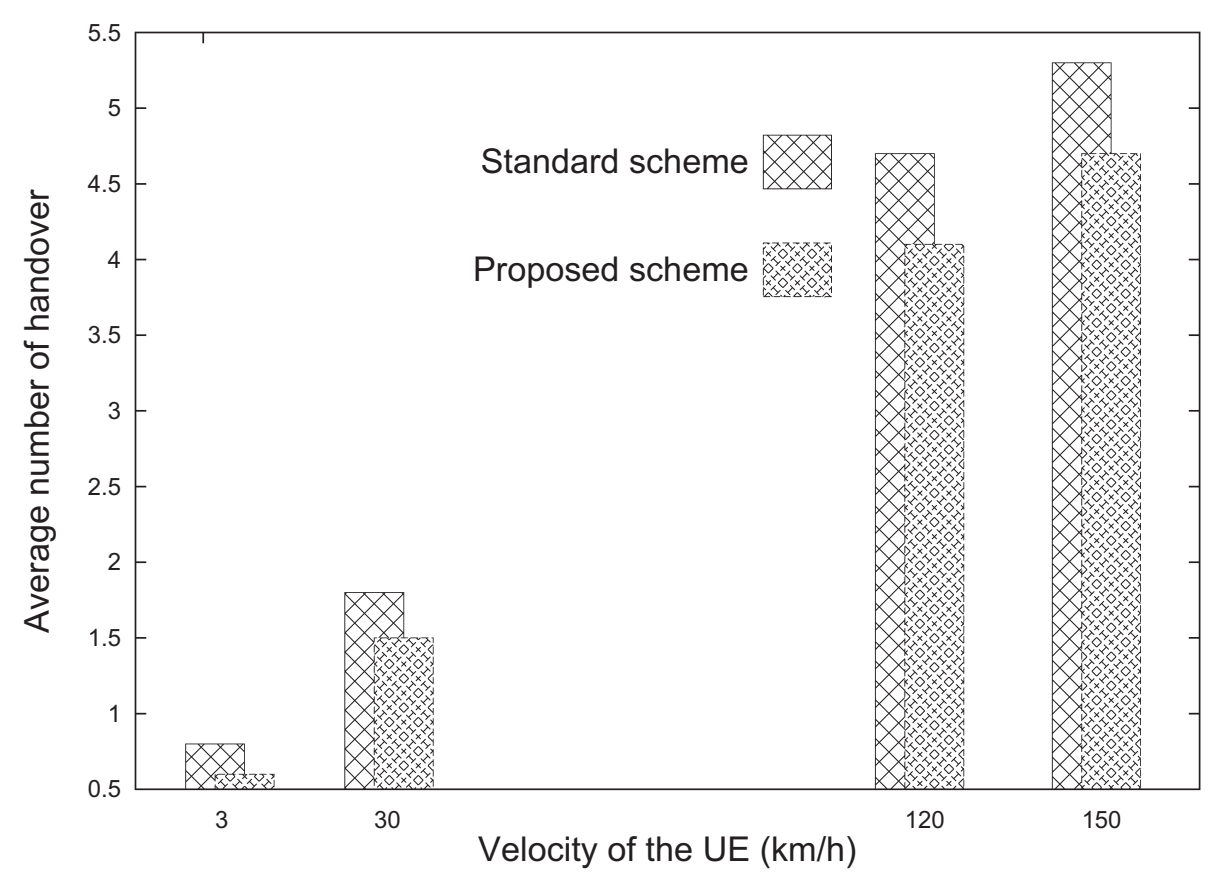

Figure 6 The average number of handovers for different handover schemes. By selecting a proper target eNB for handover with our proposed scheme, unnecessary handovers are reduced. 


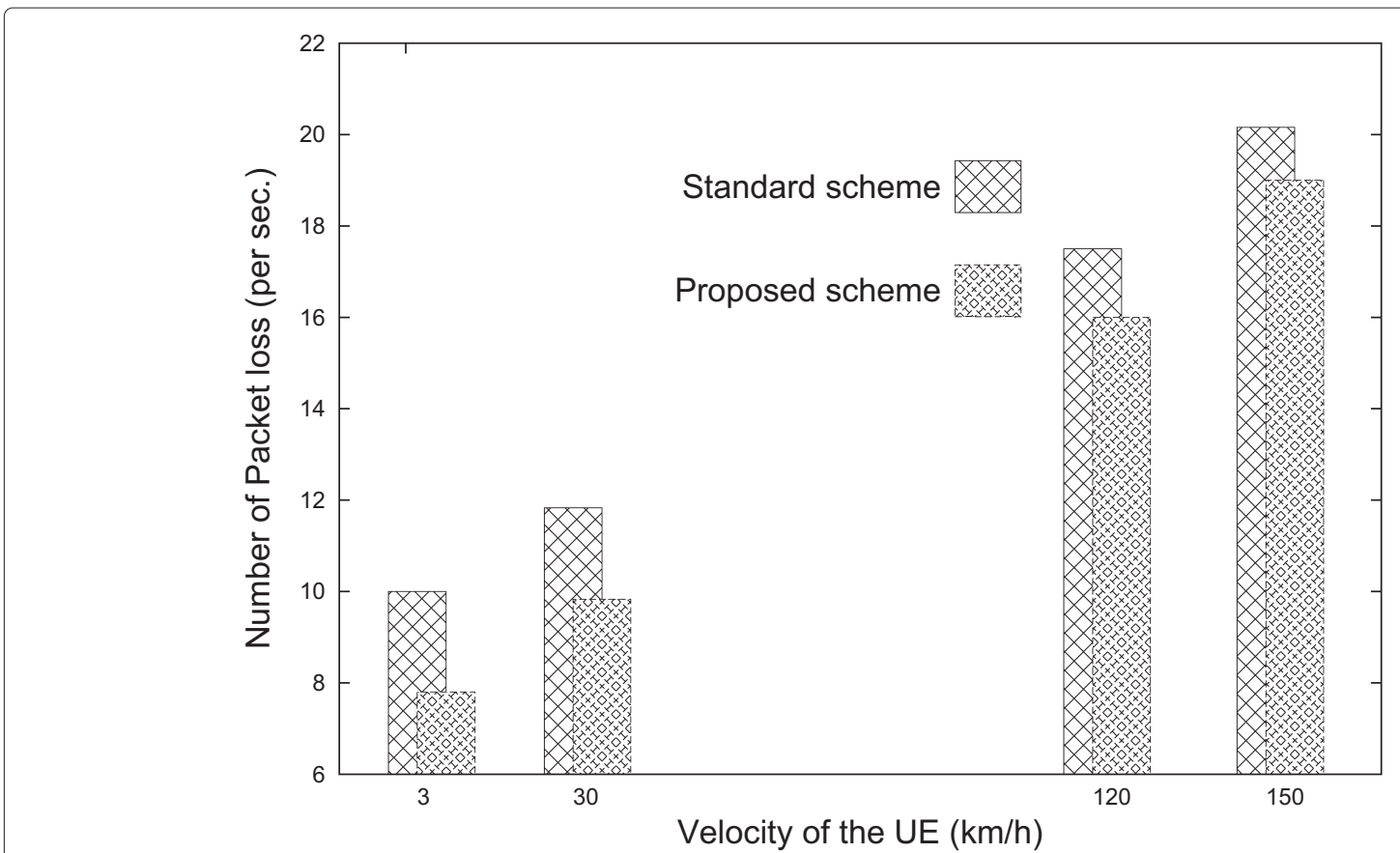

Figure 7 The number of packet loss for different handover schemes. By selecting a proper target eNB for handover with our proposed scheme, packet loss is reduced.

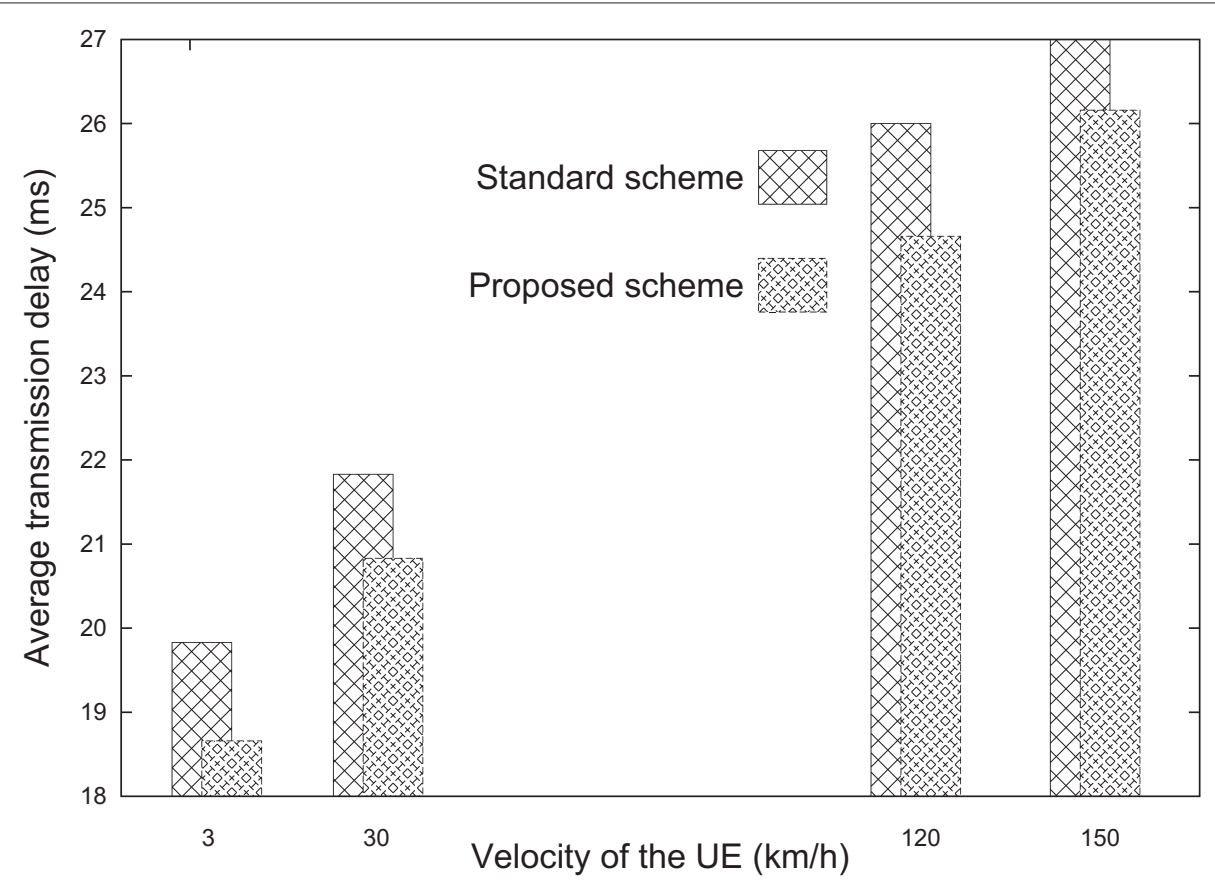

Figure 8 The average transmission delay for different handover schemes. Extra signal overhead is able to be saved with our proposed scheme, since UEs are able to connect with a proper target eNB, which results in lower transmission delay than that in the standard scheme. 
$M$ and $H$ represent the number of measurement report and handover, respectively, that the number of $N$ UEs counted within a $T$ (minutes) simulation time. $P_{s}$ and $P_{r}$ are the number of transmitted and received packets, respectively. $T_{i}^{r}$ and $T_{i}^{s}$ indicate the time the $i$ th packet received and send, respectively.

\section{Simulation results}

The simulation results are shown from Figures 5, 6, 7, and 8 . Figure 5 shows the average number of measurement reports. It is observed that the velocity of UEs will not affect the number of measurement report with a standard scheme, because it adopts a fix-duration report strategy. However, our proposed scheme has a very low number of measurement report than the standard scheme. The main reason for this is due to the fact that UE only reports its current position to its serving eNB periodically in our scheme. The serving eNB then executes our proposed direction-assisted handover procedure to select one target eNB for the UE. UE periodically measures the single strength of serving and target eNBs, instead of measuring all its neighbor eNBs.

Figures 6, 7, and 8 shows the average number of handover, number of packet loss, and average transmission delay, when UEs move with various velocities. It is observed that the average number of handovers in high velocity is higher than that in low velocity. This is because the higher the velocities of UEs, the longer the distance that UEs move, thereby increasing the number of handovers. By selecting a proper target eNB for handover, unnecessary handovers and packet loss are reduced as shown in Figures 6 and 7, since UEs are able to connect with a proper target eNB, avoiding the ping-pong effect, in which UEs move out/in its serving eNB in a short time. Extra signal overhead is able to be saved with our proposed scheme, which results in lower transmission delay than that in the standard scheme as shown in Figure 8. Therefore, our proposed scheme has outperformed than the standard scheme.

\section{Conclusions}

There is a risk of the handover fail which may result in a radio link failure in LTE networks, because it adopts a hard handover scheme. System performance is being aggravated by unnecessary handovers. It leads to transmission delay, packet loss, and signal overhead, which may seriously affect the performance of a real-time application.

In this paper, a direction prediction mechanism for LTE networks has been proposed in order to lower the unnecessary handover. We first proposed a direction prediction scheme with a simple cosine function to predict the moving direction of UEs. The eNBs, in front of the moving direction of UEs, are designated candidate eNBs for handover. Then, a target eNB is selected from the candidate eNBs for handover through an angle-based dynamic weight adjustment scheme. Simulation results revealed that under various velocities of UEs, our proposed handover scheme apparently outperforms the standard scheme from the aspect of average handover times, number of packet loss, and average transmission delay. Average handover times (17\%) can be reduced through a proper target eNB selection; hereby, $12 \%$ average number of packet loss and 5\% average packet delay time are able to be reduced. The number of measurement reports with our proposed scheme which approaches $73 \%$ can be reduced. It reflects that more power engine of UEs can be saved.

\section{Competing interests}

The authors declare that they have no competing interests.

\section{Author details}

${ }^{1}$ Department of Computer Science and Engineering, National Chung Hsing University, 250 Kuo Kuang Rd., 40227 Taichung, Taiwan. ${ }^{2}$ Department of Finance, Chaoyang University of Technology, 168, Jifeng E. Rd., Wufeng District, 41349 Taichung, Taiwan.

Received: 24 July 2014 Accepted: 4 November 2014

Published: 15 November 2014

\section{References}

1. 3GPP, 3GPP TS 36.211 Evolved Universal Terrestrial Radio Access (E-UTRA) physical channels and modulation (release 10) (European Telecommunications Standards Institute (ETSI), Sophia-Antipolis)

2. JM Chang, Z Abichar, C-Y Hsu, WiMAX or LTE: who will lead the broadband mobile internet? IT Professional. 12(3), 26-32 (2010)

3. S Srikanth, PA Murugesa Pandian, X Fernando, Orthogonal frequency division multiple access in WiMAX and LTE: a comparison. IEEE Commun. Mag. 50(9), 153-161 (2012)

4. A Ahmad, N Shah, A joint resource optimization and adaptive modulation framework for uplink single-carrier frequency-division multiple access systems. Int. J. Comm. Syst (2013). doi:10.1002/dac.2677

5. 3GPP, 3GPP TS 36.331 Evolved Universal Terrestrial Radio Access (E-UTRA) Radio Resource Control (RRC); protocol specification (release 11) (European Telecommunications Standards Institute, Sophia-Antipolis, 2012)

6. HG Myung, J Lim, D Goodman, Single carrier FDMA for uplink wireless transmission. IEEE Veh. Tech. Mag. 1(3), 30-38 (2006)

7. S-U-R Qureshi, SJ Nawaz, M Patwary, M Abdel-Maguid, A Kamar, in 2010 International Conference on Wireless Communications and Signal Processing (WCSP). The impact of propagation environment and velocity on the handover performance of LTE systems (Suzhou, 21-23 Oct 2010), pp. 1-5

8. K Dimou, M Wang, Y Yang, M Kazmi, A Larmo, J Pettersson, W Muller, Y Timner, in 2009 IEEE 70th Vehicular Technology Conference Fall (VTC 2009-Fall). Handover within 3GPP LTE: design principles and performance (Anchorage, 20-23 Sept 2009), pp. 1-5

9. H-D Bae, B Ryu, N-H Park, in Australasian Telecommunication Networks and Applications Conference (ATNAC), 2011. Analysis of handover failures in LTE femtocell systems (Melbourne, 9-11 Nov 2011), pp. 1-5

10. C-W Chang, Y-H Lin, R-H Jan, C Chen, in 2012 International Conference on Selected Topics in Mobile and Wireless Networking (iCOST). Efficient measurement procedure for handover in LTE femtocell networks (Avignon, 2-4 July 2012), pp. 119-123

11. L Luan, M Wu, Y Chen, X He, C Zhang, in IET International Conference on Communication Technology and Application (ICCTA 2011). Handover parameter optimization of LTE system in variational velocity environment (Beijing, 14-16 Oct 2011), pp. 395-399

12. M Ding, D Lopez-Perez, R Xue, AV Vasilakos, W Chen, in 2014 IEEE International Conference on Communications (ICC). Small cell dynamic TDD transmissions in heterogeneous networks (Sydney, 10-14 June 2014), pp. 4881-4887 
13. X Wang, A Vasilakos, M Chen, Y Liu, T Kwon, A survey of green mobile networks: opportunities and challenges. Mobile Network Appl. 17(1), 4-20 (2012)

14. D Lopez-Perez, X Chu, AV Vasilakos, H Claussen, Power minimization based resource allocation for interference mitigation in OFDMA femtocell networks. IEEE J. Sel. Area Comm. 32(2), 333-344 (2014)

15. D Lopez-Perez, X Chu, AV Vasilakos, H Claussen, On distributed and coordinated resource allocation for interference mitigation in self-organizing LTE networks. IEEE/ACM Trans Netw. 21(4), 1145-1158 (2013)

16. H Zhang, W Ma, W Li, W Zheng, X Wen, C Jiang, in 2011 IEEE 73rd Vehicular Technology Conference (VTC Spring). Signalling cost evaluation of handover management schemes in Ite-advanced femtocell (Budapest, 15-18 May 2011), pp. 1-5

17. C-C Lin, K Sandrasegaran, HAM Ramli, R Basukala, Optimized performance evaluation of LTE hard handover algorithm with average RSRP constraint. CoRR, 1-1 (2011)

18. W Luo, X Fang, M Cheng, X Zhou, in 2011 Fifth International Workshop on Signal Design and Its Applications in Communications (IWSDA). An optimized handover trigger scheme in LTE systems for high-speed railway (Guilin, 10-14 Oct 2011), pp. 193-196

19. X Yan, YA Sekercioglu, N Mani, in 2008 International Symposium on a World of Wireless, Mobile and Multimedia Networks, 2008. WoWMoM 2008. A method for minimizing unnecessary handovers in heterogeneous wireless networks (23-26 June 2008, pp. 1-5

20. M Anas, FD Calabrese, PE Mogensen, C Rosa, KI Pedersen, in IEEE 65th Vehicular Technology Conference, 2007. VTC2007-Spring. Performance evaluation of received signal strength based hard handover for UTRAN LTE Dublin, 22-25 Apr 2007), pp. 1046-1050

21. Y-S Chen, T-Y Juang, Y-T Lin, A secure relay-assisted handover protocol for proxy mobile IPv6 in 3GPP LTE systems. Wireless Pers Comm. 61(4), 629-656 (2011)

22. Y Lee, B Shin, J Lim, D Hong, in 2010 16th Asia-Pacific Conference on Communications (APCC). Effects of time-to-trigger parameter on handover performance in son-based LTE systems (Auckland, 31 Oct-3 Nov 2010), pp. 492-496

23. T-H Kim, Q Yang, J-H Lee, S-G Park, Y-S Shin, in 2007 IEEE 66th Vehicular Technology Conference, 2007. VTC-2007 Fall. A mobility management technique with simple handover prediction for 3 G LTE systems (Baltimore, 30 Sept-3 Oct 2007), pp. 259-263

24. $\mathrm{H} \mathrm{Ge}, \mathrm{X}$ Wen, W Zheng, Z Lu, B Wang, in International Symposium on Computer Network and Multimedia Technology, 2009. CNMT 2009. A history-based handover prediction for LTE systems (Wuhan, 18-20 Dec 2009), pp. 1-4

25. X Yan, YA Sekercioglu, N Mani, in 2008 International Symposium on a World of Wireless, Mobile and Multimedia Networks, 2008. WoWMoM 2008. A method for minimizing unnecessary handovers in heterogeneous wireless networks (Newport Beach, 23-26 June 2008), pp. 1-5

26. Y-S Huang, F-Y Leu, J-C Liu, Y-L Huang, WC-C Chu, in 2013 Eighth International Conference on Broadband and Wireless Computing Communication and Applications (BWCCA). A handover scheme for LTE wireless networks under the assistance of GPS (Compiegne, 28-30 Oct 2013), pp. 399-403

27. G Piro, LA Grieco, G Boggia, F Capozzi, P Camarda, Simulating LTE cellular systems: an open-source framework. IEEE Trans. Veh. Tech. 60(2), 498-513 (2011)

28. 3GPP TR 36.942 Evolved Universal Terrestrial Radio Access (E-UTRA); radio frequency (RF) system scenarios (European Telecommunications Standards Institute, Sophia-Antipolis, 2012)

doi:10.1186/1687-1499-2014-190

Cite this article as: Wang et al:: A moving direction prediction-assisted handover scheme in LTE networks. EURASIP Journal on Wireless

Communications and Networking 2014 2014:190.

\section{Submit your manuscript to a SpringerOpen ${ }^{\circ}$ journal and benefit from:}

- Convenient online submission

- Rigorous peer review

- Immediate publication on acceptance

- Open access: articles freely available online

- High visibility within the field

- Retaining the copyright to your article

Submit your next manuscript at $\boldsymbol{\wedge}$ springeropen.com 